\title{
Ecological Interactions of Bark Beetles with Host Trees
}

\author{
John A. Byers \\ US Arid-Land Agricultural Research Center, ARS USDA, 21881 North Cardon Lane, Maricopa, AZ 85138, USA \\ Correspondence should be addressed to John A. Byers, john.byers@ars.usda.gov \\ Received 9 October 2012; Accepted 9 October 2012 \\ Copyright (c) 2012 John A. Byers. This is an open access article distributed under the Creative Commons Attribution License, \\ which permits unrestricted use, distribution, and reproduction in any medium, provided the original work is properly cited.
}

Certain species of bark beetles in the insect order Coleoptera, family Curculionidae (formerly Scolytidae), are keystone species in forest ecosystems. However, the tree-killing and wood-boring bark and ambrosia beetles are also among the most damaging insects of forest products including lumber, paper, and ornamental/recreational trees. The pest status of these beetles has been elevated with the advance of global warming and moderate to severe area-wide droughts, exacerbated by mismanagement and prevention of fires over decades. The ecology and chemical ecology of bark beetles has been and still is an exciting area of research, particularly since bark beetles utilize a wide array of semiochemicals in communication and in interactions with plants. Bark beetle chemical ecology is intimately connected and intertwined with behavioral and physiological processes that are still largely unknown in many species. Development of more efficient pest management practices will require a much deeper understanding of the ecology of bark beetles facilitated by interdisciplinary observations and experiments on many levels. Potential topics for this special issue include host-tree finding and selection, resistance by the tree, avoidance of tree defenses, insect/tree microbial associations, regulation of colonization density, ecology of predators and parasitoids, communication, biosynthesis of semiochemicals, behavioral assays and antennal responses, population management, models of dispersal and trapping, and reviews. Many of these topics and others are covered in part in the 12 articles in the special issue on "Bark beetle ecology and interactions with host trees."

F. Schlyter in his article "Semiochemical diversity in practise: Antiattractant semiochemicals reduce bark beetle attacks on standing trees - a first meta-analysis" brings together the results of 33 recent studies on attractive traps and repellent chemicals (verbenone and/or nonhost tree volatiles) designed to reduce bark beetle colonization of host trees. He found using Cohen's effect size that the use of repellent chemicals caused a significant reduction in attacked and killed trees in most studies. The results were not affected by publication year and the plots indicated there were little bias in reporting of only those studies showing effects of repellents. The use of natural repellents at higher dosages can allow stressed trees to survive by protecting themselves with their innate defenses. He recommends more precise reporting of results, more unified experimental designs, and further meta-analyses that include "grey literature" and more beetle species.

S. D. Reay et al. in their article "Hylastes ater (Curculionidae: Scolytinae) affecting Pinus radiata seedling establishment in New Zealand" report on the pine bark beetle Hylastes ater that was introduced into New Zealand about 100 years ago. In the 1950-1970s, biological control was attempted with limited success, and now there is renewed interest in developing a better understanding of the pest status on seedlings and to evaluate the role of the beetle in vectoring sapstain fungi in order to develop options for management. A number of findings relevant to the New Zealand exotic forest industry are presented that reveal the role of secondary bark beetles.

A. Angst et al., Rüegg, and Forster report in their article "Declining bark beetle densities (Ips typographus, Coleoptera: Scolytinae) from infested Norway spruce stands and possible implications for management" that eight-toothed spruce bark beetles (Ips typographus) during the last 20 years have killed millions of cubic meters of standing spruce in central Europe. Beetle densities were monitored using pheromone traps along transects from infested stands to spruce-free buffer zones. The beetle densities decreased rapidly with increasing distance from the infested spruce stands, falling below high risk thresholds within a few hundred meters from the infested stands. The decrease in catches was more pronounced in open land and in an urban area than in a broadleaf stand. Buffer zones without spruce of $500 \mathrm{~m}$ width can probably reduce densities of spreading beetles. 
Several articles deal with pine shoot beetles of the genus Tomicus; for example, T. Zhao and B. Långström in their article "Performance of Tomicus yunnanensis and Tomicus minor (Col., Scolytinae) on Pinus yunnanensis and Pinus armandii in Yunnan, southwestern China" report that $T$. yunnanensis and Tomicus minor have caused substantial tree mortality to Yunnan pine (Pinus yunnanensis) in southwestern China, whereas the beetles rarely attack Armand pine (Pinus armandii). The suitability of $P$. armandii as host material for the two Tomicus species was tested by introduction of the beetles to branches and logs of the two pines during shoot feeding and trunk attack phases. Successful shoot feeding was observed by both species on both host trees, but the performance of both Tomicus species was much better on $P$. yunnanensis than on $P$. armandii. In the laboratory, T. yunnanensis and T. minor produced similar progeny in both pine species' logs, but the brood beetles emerging from Armand pine weighed less than those from Yunnan pine. Thus, $P$. armandii may be a potential host for T. yunnanensis and T. minor,but more experiments should assess the risk of these insects to stands of $P$. armandii.

In the first of two articles by R. C. Lu et al. on the "Attraction of Tomicus yunnanensis (Coleoptera: Scolytinae) to Yunnan pine logs with and without periderm or phloem: an effective monitoring bait," the authors report experiments with host log baits to develop a pest monitoring system using host tree kairomone. Yunnan pine logs with peeled-off periderm (outer bark) with sticky adhesive areas captured significantly more beetles than on untreated control logs with adhesive. T. yunnanensis fly mostly during the afternoon according to trap catches. Attraction to the periderm-peeled logs decreased considerably when they were peeled further to remove the phloem, indicating that phloem volatiles play a role in selection of the host by the beetle. The readily available log baits appear useful for monitoring pine shoot beetle populations in integrated pest management programs. In a second paper by these authors, "Coexistence and competition between Tomicus yunnanensis and T. minor (Coleoptera: Scolytinae) in Yunnan pine," they found that T. yunnanensis initiated dispersal from pine shoots to trunks in November, while the majority of T. minor begin to transfer in December. The patterns of attack densities of these two species were similar, but with T. yunnanensis colonizing the upper section of the trunk and T. minor the lower trunk. The highest attack density of T. Yunnanensis was $297 \mathrm{egg}$ galleries $/ \mathrm{m}^{2}$, and the highest attack density of T. minor was 305 egg galleries $/ \mathrm{m}^{2}$. Although there was significant overlap for the same bark areas, the two species generally colonize different areas of the tree, which reduces the intensity of competition for the relatively thin layer of phloem-cambium tissues where the beetles feed and reside.

Q.-H. Zhang et al. in their article "2-Methyl-3-buten2-ol, a pheromone component of conifer bark beetles found in the bark of nonhost deciduous trees" report that volatiles from the bark of aspen, Populus tremula and two species of birch (Betula pendula and B. pubescens), were collected from both newly cut bark chips and undamaged stems and subjected to combined gas chromatography and mass spectrometry (GC-MS) analysis. The results showed the presence of 2-methyl-3-buten-2-ol (MB), one of the two principal aggregation pheromone components of the spruce bark beetle, Ips typographus, in samples of all three tree species. In addition, an oxygenated hemiterpene, 3-methyl-3-buten-2-one, and (E)-3-penten-2-ol were collected. Only trace amounts of MB were detected in some aeration samples of the fresh bark chips, and no $\mathrm{MB}$ was found from the aeration samples of undamaged stems. The occurrence of $\mathrm{MB}$ was also confirmed in the bark of four exotic birch species: B. albo-sinensis, $B$. ermanii, B. jacquemontii, and B. maximowicziana, but not in the European pines/spruces and the common yeasts. The results raise major questions regarding the evolution, the biosynthesis, the tropospheric chemistry, and the ecological role of $\mathrm{MB}$.

G. Birgersson et al. report on the "Pheromone production, attraction, and interspecific inhibition among four species of Ips bark beetles in the southeastern USA." Hindgut volatiles from attacking, unmated males of Ips avulsus, I. calligraphus, I. grandicollis, and I. pini were analyzed by GC-MS and these results were used to formulate baits for the four bark beetle species. The bioassays were subtractive for the compounds identified in the hindgut analysis of each species, and volatiles identified in sympatric species were added as potential inhibitors alone and in combination. The subtractive assays showed that I. grandicollis and I. calligraphus share (-)-(4S)-cis-verbenol as one pheromone component. The second synergistic pheromone component of I. grandicollis, $(-)$-(S)-ipsenol, acts as an interspecific inhibitor of I. calligraphus, while the second synergistic pheromone component of $I$. calligraphus, $( \pm)$-ipsdienol, acts as an interspecific inhibitor to I. grandicollis. I. avulsus and I. pini were found to be very similar in hindgut volatiles, and both use ipsdienol and lanierone as synergistic pheromone components. Lanierone was found to be an interspecific inhibitor for both I. calligraphus and I. grandicollis.

J. A. Byers reports that "Bark beetles, Pityogenes bidentatus, orienting to aggregation pheromone avoid conifer monoterpene odors when flying but not when walking." Previous studies and data in this paper suggest that odors from healthy host Scotch pine (Pinus sylvestris) and nonhost Norway spruce (Picea abies), as well as major monoterpenes of these trees at natural release rates, significantly reduce the attraction of flying bark beetles, Pityogenes bidentatus, of both sexes to their aggregation pheromone components grandisol and cisverbenol in the field. In contrast, $P$. bidentatus males and females walking in an open-arena olfactometer in the laboratory did not avoid monoterpene vapors at release rates spanning several orders of magnitude in combination with aggregation pheromone. This apparent contradiction can be explained if the bark beetle avoids monoterpenes when flying as a mechanism for avoiding nonhost species, vigorous, and thus unsuitable host trees, as well as harmful resinous areas of hosts. After landing, inhibition of this flight avoidance response in beetles would allow them to initiate, or to find and enter, gallery holes with high monoterpene vapor concentrations in order to feed and reproduce.

J. A. Byers and G. Birgersson write about "Host-tree monoterpenes and biosynthesis of aggregation pheromones in the bark beetle Ips paraconfusus." In the 1970-80s, a paradigm 
developed that Ips bark beetles utilize the host tree's preformed monoterpene myrcene as a precursor that is simply hydroxylated in order to minimize pheromone (ipsenol and ipsdienol) biosynthetic costs. In 1990, however, amounts of ipsenol and ipsdienol produced by male I. paraconfusus feeding in five host pine species were found to be very similar, even though GC-MS showed that there was no detectable myrcene precursor in one of the species of pine (Pinus sabiniana). Subsequent research showed that ipsenol and ipsdienol are biosynthesized from smaller precursors such as acetate and mevalonate, and this de novo pathway is the major one, while host tree myrcene conversion by the beetle is the minor one. Concentrations of myrcene, $\alpha$-pinene, and other major monoterpenes in five pine hosts (Pinus ponderosa, $P$. lambertiana, $P$. jeffreyi, $P$. sabiniana, and $P$. contorta) of $I$. paraconfusus are reported and a scheme for biosynthesis of ipsdienol and ipsenol from myrcene and possible metabolites such as ipsenone is presented. Coevolution of bark beetles and host trees is discussed in relation to pheromone biosynthesis, host plant selection/suitability, and plant resistance.

The issue deals with theoretical topics regarding evolutionary selection for host tolerance and choice in the paper by J. A. Byers "A population genetic model of evolution of hostmate attraction and nonhost repulsion in a bark beetle Pityogenes bidentatus." Studies have shown that the bark beetle Pityogenes bidentatus avoids volatiles of nonhost trees (Norway spruce, birch, and oak) and healthy host Scotch pine when orienting to aggregation pheromone. A population genetic model of two behavioral genes was hypothesized where $\mathrm{AA}, \mathrm{Aa}$, and aa were allele combinations regulating orientation to host tree and pheromone odors, and $\mathrm{BB}, \mathrm{Bb}$, and $\mathrm{bb}$ were combinations allowing avoidance of nonhost and unsuitable host odors. The nine possible genotypes were assigned different survival factors that remained constant during simulation. The initial proportion of aabb genotype (little aggregation/host response and little avoidance of nonhosts) was $\sim 1.0$ when a mutation was hypothesized that caused better orientation to host/beetle odors (Aabb) and another mutation causing more efficient avoidance of nonhosts $(\mathrm{aaBb})$. After these initial mutations, the model used indiscriminate mating of genotypic proportions and subsequent survival as input for each successive generation. The results indicate that $\mathrm{AABB}$ eventually fixates in the populations in some scenarios, while AABB and other genotypes reach stable equilibriums in other models depending on genotypic survival values supported by ecologically sound assumptions. The models indicate how the development of insecticide resistance in pest insects as well as host selection and tolerance may proceed.

M. N. Andersson in a comprehensive review "Mechanisms of odor coding in coniferous bark beetles: from neuron to behavior and application" discusses how coniferous bark beetles locate their hosts by means of olfactory signals, such as pheromones, and host- and non-host compounds. Behavioral responses to these volatiles are well known, but apart from the olfactory receptor neurons (ORNs) detecting pheromones, information on the peripheral olfactory physiology has been limited until recently. Several new classes of ORNs for the spruce bark beetle, Ips typographus, have been described and the odor encoding mechanisms and links between behavioral responses and ORNs responses were investigated, allowing for a more profound understanding of bark beetle olfaction. This paper reviews the physiology of bark beetle ORNs and their relevance in a behavioral context focusing on I. typographus.

While the articles are limited in number they do address a wide variety of topics regarding bark beetle interactions directly with the host tree as well as with other colonizing bark beetles in the host tree or during the process of finding hosts and avoiding nonhost plants and bark areas with severe intra- and interspecific competition and thus maximize fitness.

John A. Byers 

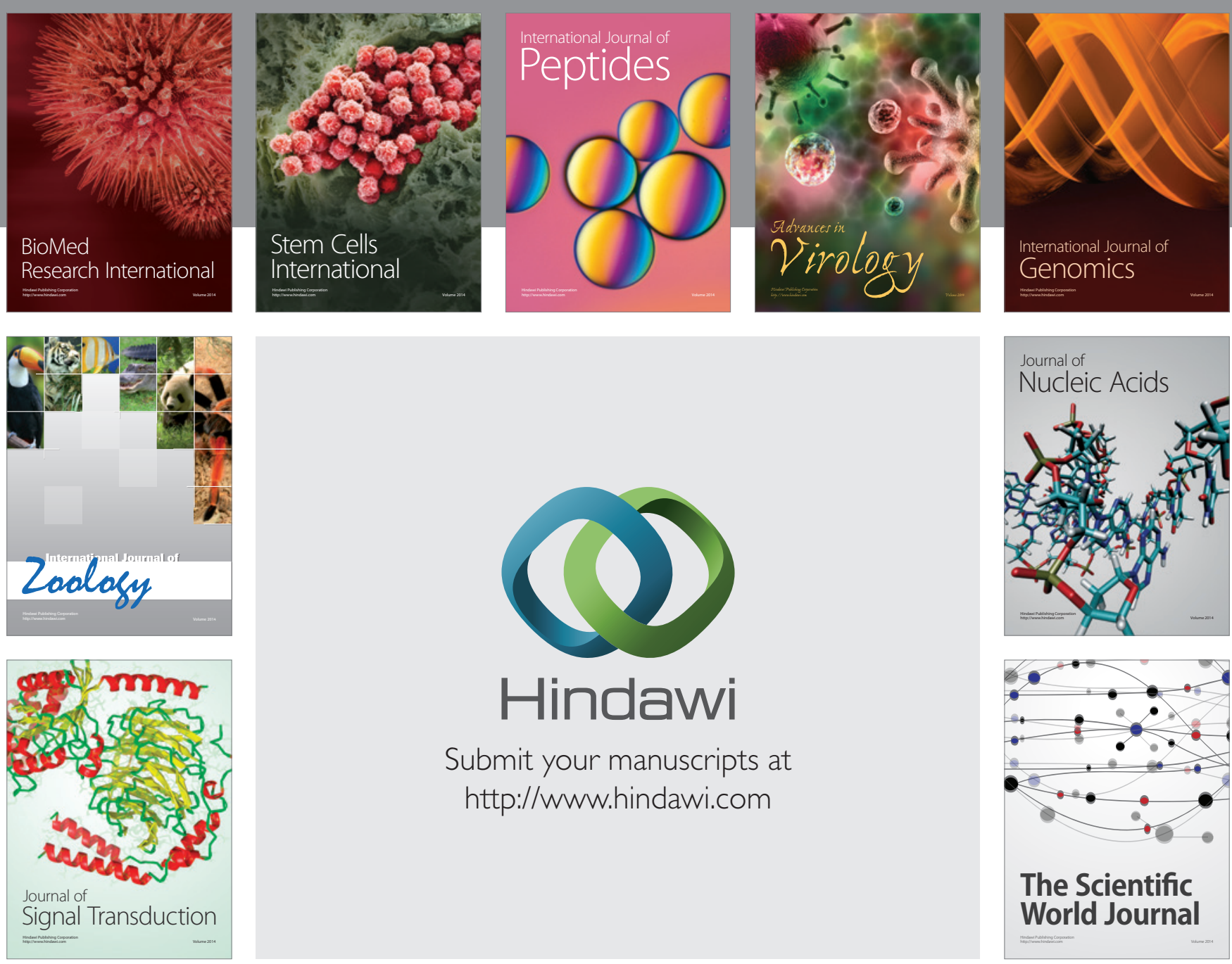

Submit your manuscripts at

http://www.hindawi.com
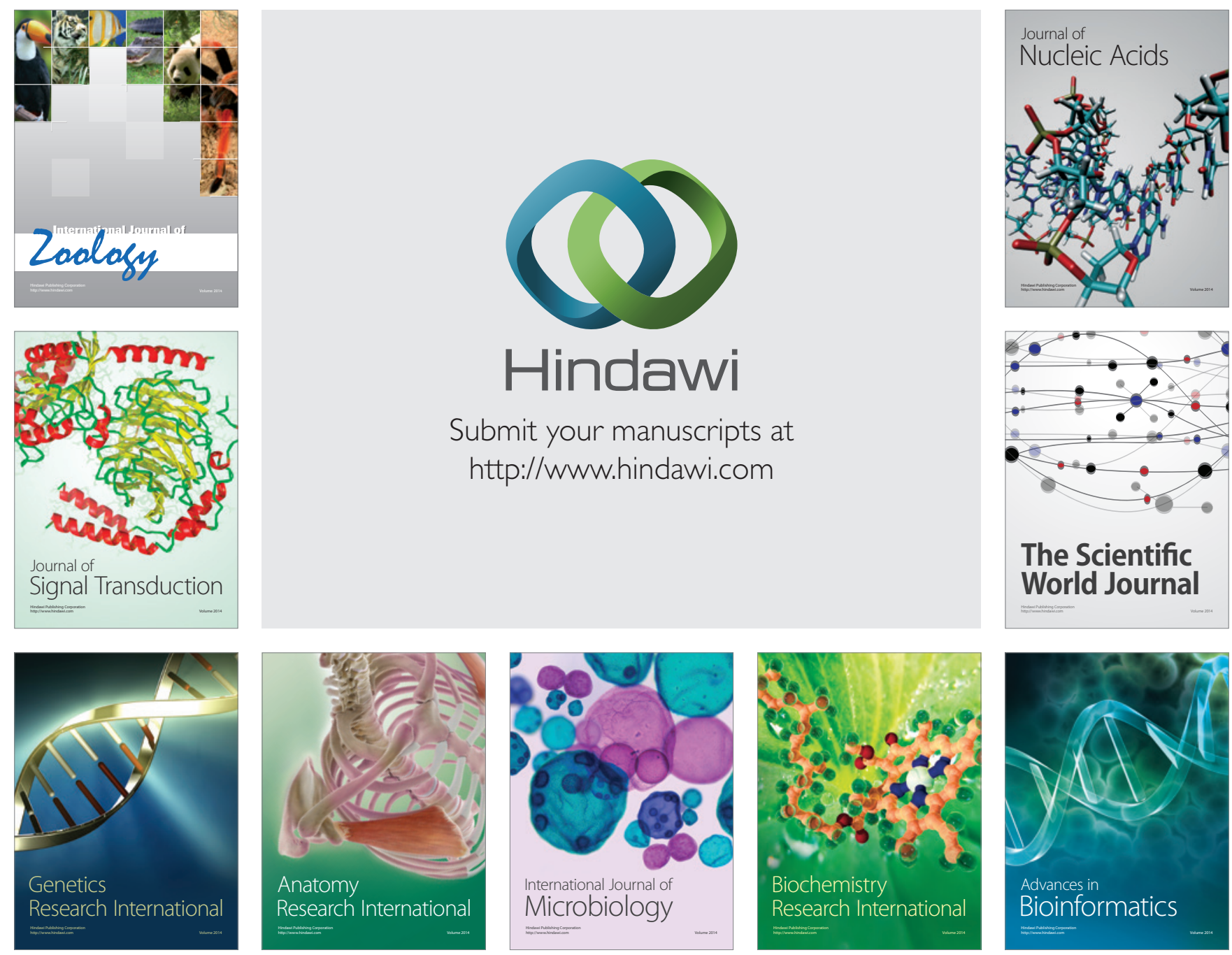

The Scientific World Journal
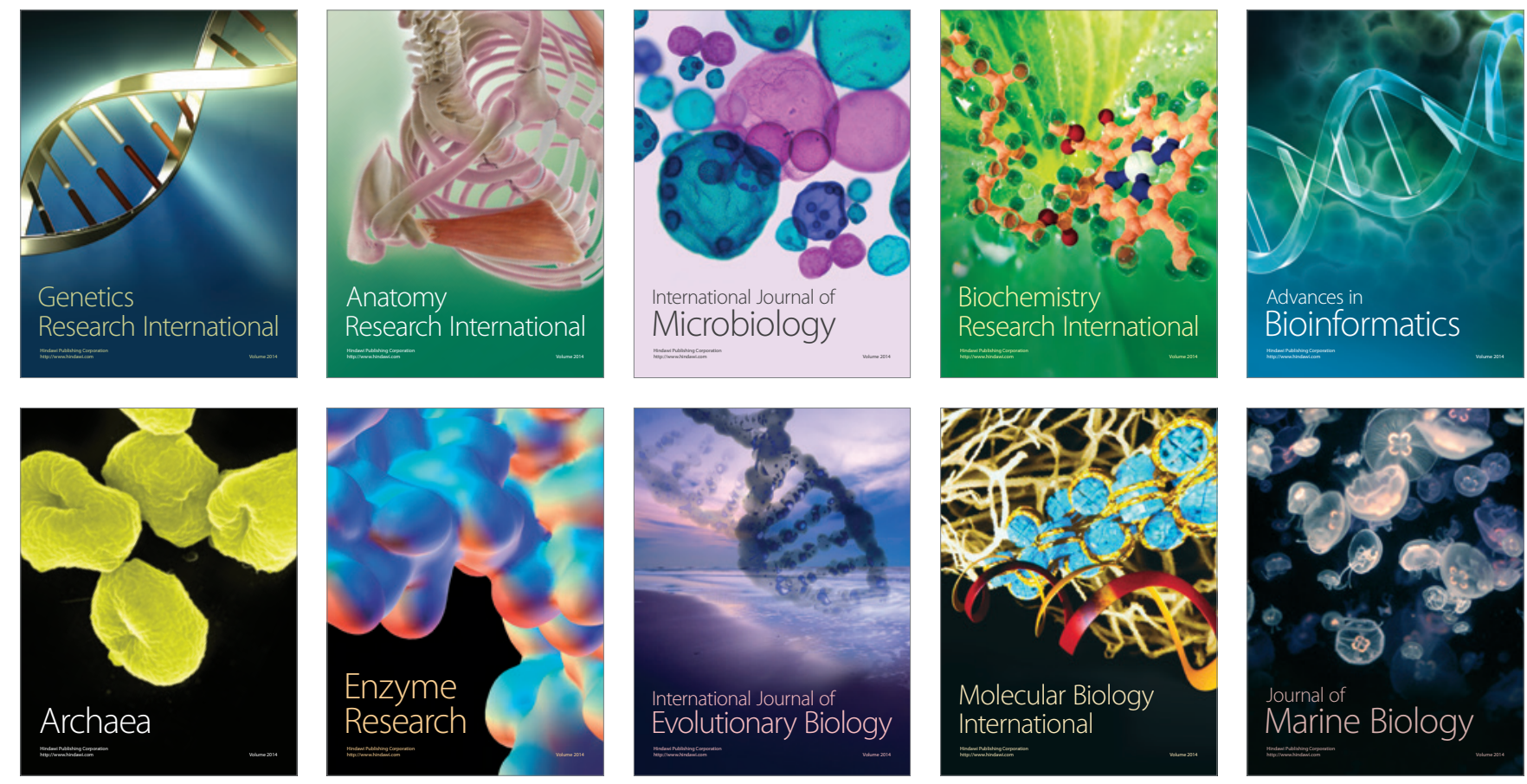